\title{
Approche agro-pédologique des zones pastorales sur calcschistes sédimentaires (Beaufortain-Alpes du Nord). II. Répartition des sols et des valeurs d'usage pastoral
}

\author{
JM Dorioz *, F Van Oort ** \\ INRA, station de sciences du sol, Versailles, France
}

(Reçu le 8 juin 1989; accepté le 10 mars 1991)

\begin{abstract}
Résumé - L'originalité de la pédogénèse sur calschistes sédimentaires, en altitude (étage subalpin), réside dans l'homgénéité du résidu de décarbonatation (plaquettes de micas de taille limons fins). Au sein des sols développés en place, la principale évolution qui se manifeste entre l'altérite et la surface du sol, est une microdivision de ces particules limoneuses. Celle-ci s'accompagne d'une disparition du système de macroporosité hérité de la décarbonatation de la roche et de l'apparition, dans l'horizon (B), d'un système de micropores. II s'ensuit une faible disponibilité de

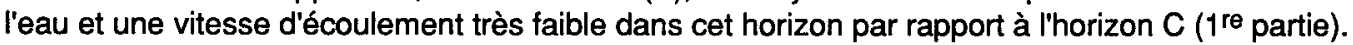

Cette organisation du sol liée à la pédogénèse, a des répercussions très significatives sur la végétation prairiale et sa dynamique. Elle représente une contrainte agronomique qui s'exprime surtout en situation de plateau. L'approvisionnement en eau et en éléments nutritifs (bases) par le système racinaire du tapis végétal, est alors limité par l'épaisseur de l'horizon (B) et les risques de dégradation de la valeur fourragère sont maximaux. L'évolution pédogénétique et les potentialités dépendent alors étroitement de l'intensité et des modalités de l'exploitation pastorale actuelle et (ou) passée, seuls facteurs susceptibles d'atténuer et de compenser les effets des propriétés physiques défavorables de la couverture pédologique. Au total, sur les plateaux le paysage pastoral porte d'abord la marque des pratiques agricoles.

Inversement, dès que la pente s'accentue (situation de versant de solifluxion), la circulation de l'eau et la topographie deviennent les facteurs prépondérants pour expliquer la différenciation en mosaïque des sols et des végétations et révéler les contraintes spécifiques d'entretien des diverses zones.
\end{abstract}

Alpes / calcschiste / pâturage / valeur pastorale / sol de montagne

Summary - Landscape ecology of alpine pastures in a sedimentary calcschist area (Beaufortain). II. Soil distribution and pastoral values. The originality of pedogenesis on calcschist in the Beaufortain mountain pasture region lies in the homogeneity with respect to the size, form and mineralogical nature of the constituents of the decarbonation residue: mica platelets with fine silt particles. In in situ developed soils, the main evolution observed between the weathering horizon and the soil surface is of a physical nature. The physical breakdown of silt particles takes place concomitantly with the disappearance of the pore system inherited from the weathered rock. In the (B) horizon, a microscopy (pore size $\approx 0.6 \mu \mathrm{m}$ ) becomes visible, which is the result of the microdivided mica particle arrangement (particle size $<6 \mu \mathrm{m}$ ). A low water availability and low hydraulic conductivities in this soil horizon with regard to the $C$ horizon therefore occurs. These data have been presented in the first paper.

Such soil structure modification produces pertinent repercussions with respect to pasture vegetation. Significant agronomic constraints thus arise in all areas with a flat topography (plateau, fig 1, table II). The uptake of water and nutritious elements (bases) by the root systems of the pasture cover is impeded as the thickness of the (B) horizon with unfavorable physical properties increases (figs 2, 3). In such an environment with a strong tendency to acidification, soil evolution is highly dependent on agronomic farming intensity, both today and in the past (table II, fig 4).

As soon as hill slopes get steeper (solifluxion slopes, fig 5, table III), the superficial water movements and topography become major factors explaining soils and vegetations differentiation, often organized in mosaic distribution patterns (table III, IV). Hence, within the same mountain pasture area, calcareous, acid and hydromorphic soils can be observed, each soil type imposing its own specific farming constraints (table IV).

Alps / calcschist / pasture / pastoral value / mountain soils

Adresses actuelles: *INRA, science du sol, institut de limnologie, BP 511, 74203 Thonon-les-Bains cedex;

** CRAAG, station agroclimatique de la zone caraibes, unité de science du sol, BP 1232, 97184 Pointe-à-Pitre cedex, France 


\section{INTRODUCTION}

L'étude des règles d'organisation des sols dans le paysage nous sert de base pour établir un diagnostic agronomique concernant la couverture pédologique d'une région pastorale des Alpes du Nord (le Beaufortain). La région étudiée (altitude $>1400 \mathrm{~m}$ ) est caractérisée par une forte homogénéité du support géologique (calschistes sédimentaires ou calcshales). Cette situation permet de bien définir le rôle de paramètres du milieu tels que le pendage (Dejou et al, 1971), ou la circulation de l'eau. Elle permet aussi d'interpréter les interactions pédogénèse-morphogénèse (Tricart et Killian, 1979), et pédogénèse - exploitation agricole.

Dans une première partie (Van Oort et Dorioz, 1991) nous avons analysé les propriétés spécifiques que confèrent aux sols développés en place, la nature du résidu d'altération des calcschistes. En bref, la décarbonatation libère des constituants minéraux très homogènes en terme de dimension (limons fins), de forme (plaquettes à faible surface externe, donc faible (EEC) et de nature minéralogique (micro-micas). Ces caractéristiques expliquent bien la tendance à l'acidification des sols. Par ailleurs, lors de la pédogénèse, le système de porosité du matériau d'altération subit une profonde évolution. À l'horizon $C$ mince et très poreux se superpose un horizon $B$ épais, microporeux, peu perméable. II en résulte un comportement hydrique original de ces sols avec, en particulier, une sécheresse physiologique résultant des propriétés porales de B.

Cette deuxième partie se propose d'expliquer comment les propriétés physiques du matériau d'altération, combinées aux facteurs géomorphologiques, influent sur l'organisation du paysage pastoral et sur les problèmes agronomiques qui s'y rattachent (répartition des ressources fourragères et des risques de dégradation, facteurs de maîtrise de la végétation fourragère).

Cette réflexion s'appuie essentiellement sur l'étude comparée du fonctionnement de secteurs de référence, en terme de relations solsvégétations-exploitations. Les sites choisis fournissent un bon inventaire des milieux pastoraux de la région. Le classement retenu pour les herbages (tableau I) et la caractérisation de leurs valeurs d'usage, s'appuient sur nos travaux antérieurs (Dorioz et Party, 1987; Dorioz, 1987), et sur la typologie établie par Dubost et Bornard (1989). Des informations complémentaires ont été obtenues par enquête et par expérimentation (Sabin, 1987; Reydet, 1985). II faut préciser d'emblée que les "valeurs d'usage» (Jeannin et al, 1991) des végétations pâturées de montagne dépendent moins de leur productivité, que de leur appétence et de leur aptitude à la repousse. Enfin la prise en compte des risques spécifiques de dégradation de la végétation en dessous de certains seuils d'entretien, complète ce classement des herbages.

\section{ORGANISATION GÉNÉRALE DU RELIEF ET CHOIX DES SECTEURS DE RÉFÉRENCE}

Les calcschistes représentent l'élément dominant du substrat géologique régional. Ils forment des reliefs constituant une série de montagnes avec des flancs O/NO plus abrupts et souvent marqués par des falaises. Les flancs E/SE correspondent à des versants dont le pendage de la roche est conforme, ce qui facilite les mouvements de glissement des matériaux superficiels (Nicot, 1988). Quel que soit le versant, on dénote, au-delà d'une pente moyenne de $10 \%$, une forte fréquence de la solifluxion, dynamique favorisée par l'abondance, la nature et les propriétés du résidu libéré par la décarbonatation. En effet, le matériau d'altération, très riche en limons fins, constitué de plaquettes, se sature d'eau et se réoriente facilement, ce qui le prédispose à la solifluxion (Van Oort, 1984). Par ailleurs, la pédogénèse tend à donner naissance à des horizons $(B)$ peu perméables, évolution qui facilite les déformations des couvertures pédologiques. Quand la pente faiblit la solifluxion s'atténue, puis disparaît.

Nos secteurs de référence (fig 1 in Van Oort et Dorioz, 1991) incluent des toposéquencés extrêmes. Ils couvrent par conséquent l'essentiel de la gamme de variations des conditions topographiques et géomorphologiques existant en zone pâturable sur calcshales. II s'agit :

- d'un vaste secteur de "plateau", représentatif des surfaces pastorales au relief peu accusé;

- de 2 versants en pentes fortes soumis à la solifluxion, et constituant les 2 flancs d'une même montagne pastorale.

\section{LES UNITÉS DE PLATEAU}

Le plateau (Les «Saisies", 1 500-1 800 m d'altitude) est un ensemble subhorizontal de 500 ha 


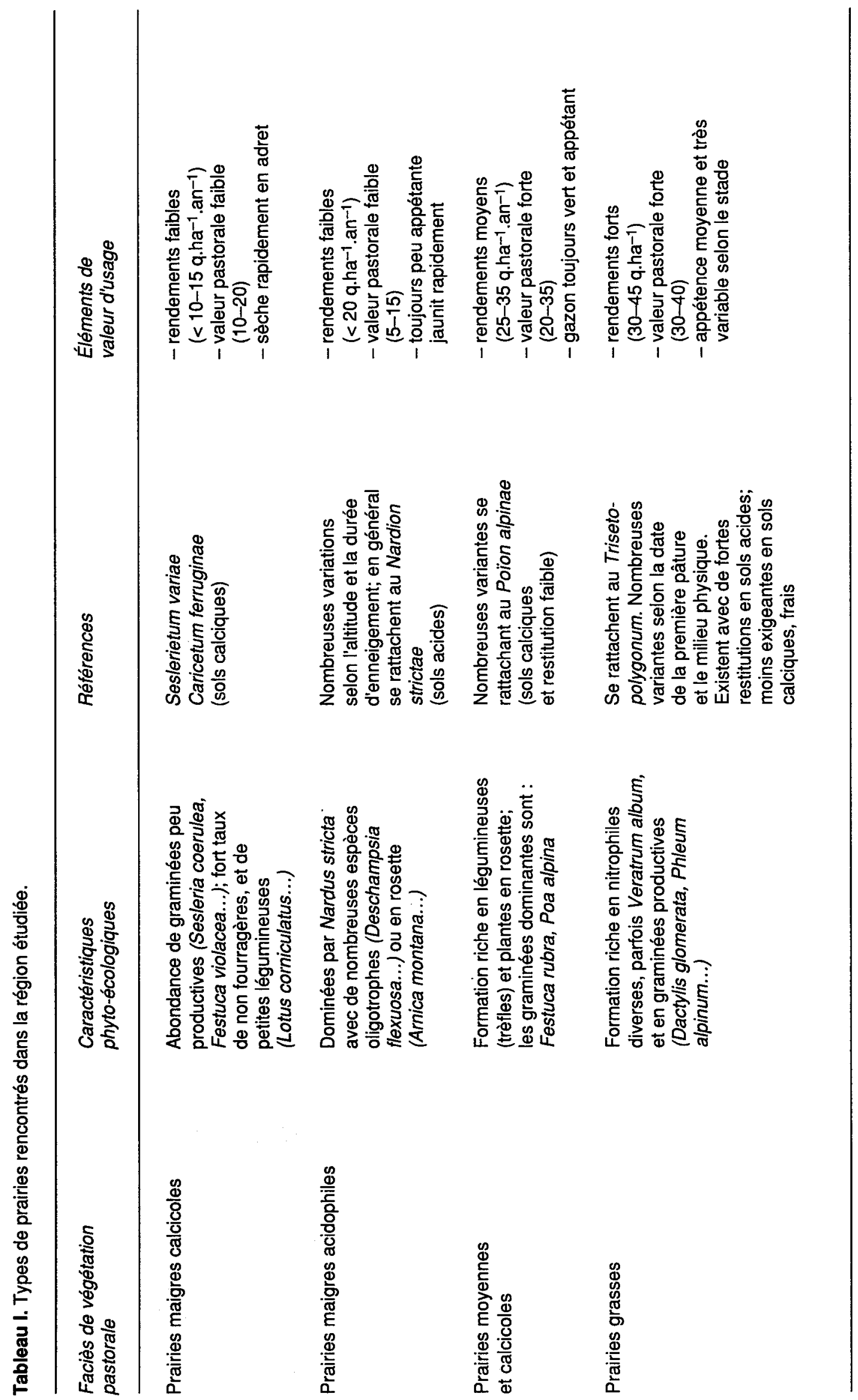


caractérisé par une couverture végétale continue à dominante herbacée, des pentes faibles $(<10 \%)$ sur des calcschistes à pendage conforme.

Localement il existe quelques petites dolines avec des sols hydromorphes complexes décrits par ailleurs (Van Oort, 1984); il s'agit de stations marginales. En fait, pour l'essentiel, la couverture pédologique est formée de sols développés en place dont la seule source de calcium est la roche mère. Celle-ci se situe pour l'essentiel à plus de $60 \mathrm{~cm}$ de profondeur ce qui, à l'étage subalpin, constitue une situation tout à fait favorable à l'acidification (Legros et al, 1980). Sous prairie maigre, le sol est alors de type sol brun acide, identique au sol de référence décrit en détail dans la première partie.

Dans un milieu physique aussi homogène, les différenciations de la couverture pédologique sont surtout liées à l'ancienneté des pratiques pastorales (fig 1), bien qu'on puisse discerner des variations très localisées, selon les profondeurs de la roche mère. Nous allons montrer comment, quelle que soit la station, les caractéristiques spécifiques du matériau d'altération conditionnement fortement les propriétés du milieu pastoral.

\section{Profondeur du sol, végétation et valeur pastorale}

L'étude de la relation entre végétation et épaisseur du sol est possible dans les stations où la roche s'enfonce très lentement par rapport à la surface. Une telle situation a fait l'objet d'un échantillonnage dense (fig 2), avec 5 stations

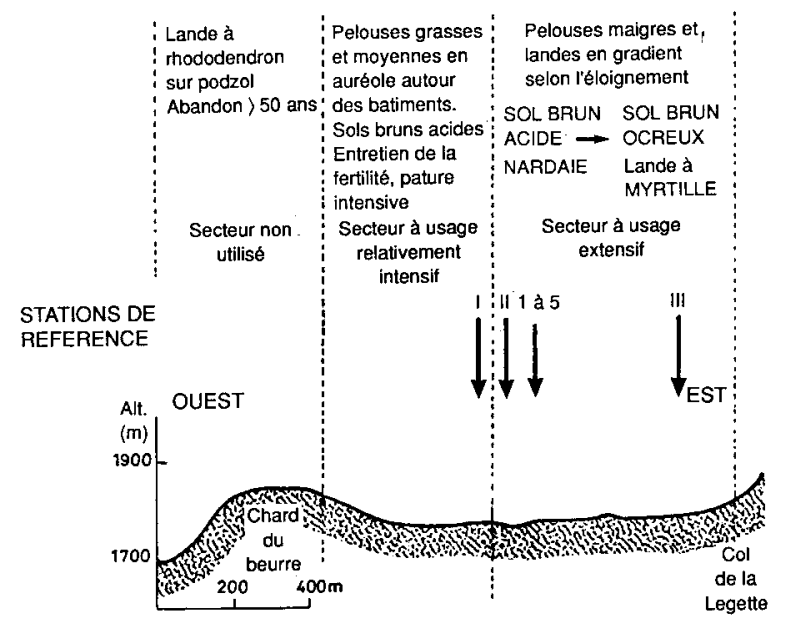

Fig 1. Le transect du plateau des Saisies : position des sols dans le paysage et différenciation agropédologique (les flèches indiquent la situation des stations types). étudiées sur un transect de 2,50 m. L'utilisation pastorale est homogène (parcours extensifs sans pratique organisée des restitutions).

Sur les 5 stations, 2 portent des végétations maigres, à valeur faible (valeur pastorale < 10), jaunissant rapidement en été :

- station 1 : sol lithocalcique superficiel $<10$ $\mathrm{cm}$ ) à flore xéro-calcicole (rattachée au type maigre calcicole)

- station 5 : sol brun acide profond $(60 \mathrm{~cm})$ à flore réputée à la fois acidophile et un peu xérophile (faciès sec du Nardion, Richard et Pautou, 1982).

Entre ces 2 extrêmes les stations 2 (sol brun eutrophe) et 3, 4 (sol brun mésotrophe) portent une flore dominée par Phleum alpinum et Alchemilla vulgaris, (apparentée au Poïon alpinae), ce qui traduit un bien meilleur approvisonnement de la pelouse en eau et en éléments nutritifs. La physionomie de cette végétation mésophile, dense, élevée, bien verte, même en été, d'assez bonne valeur pastorale (environ 30), s'oppose nettement à celle des stations situées aux extrémités de la micro-séquence (1 et 5).

Les analyses chimiques des sols montrent que cette séquence se différencie très clairement par des variations rapides du taux de saturation $(\mathrm{S} / \mathrm{T})$ des divers horizons (fig 3 ). Au fur et à mesure que le sol s'épaissit, une désaturation se manifeste, d'abord dans l'horizon B (stations 2, $3,4)$ puis dans les horizons de surface (station 5). Or, les bases sont puisées dans l'horizon $C$, horizon par ailleurs toujours riche en eau disponible (Van Oort et Dorioz, 1991). Par conséquent, l'évolution observée traduit une diminution des remontées d'éléments liées aux prélèvements racinaires.

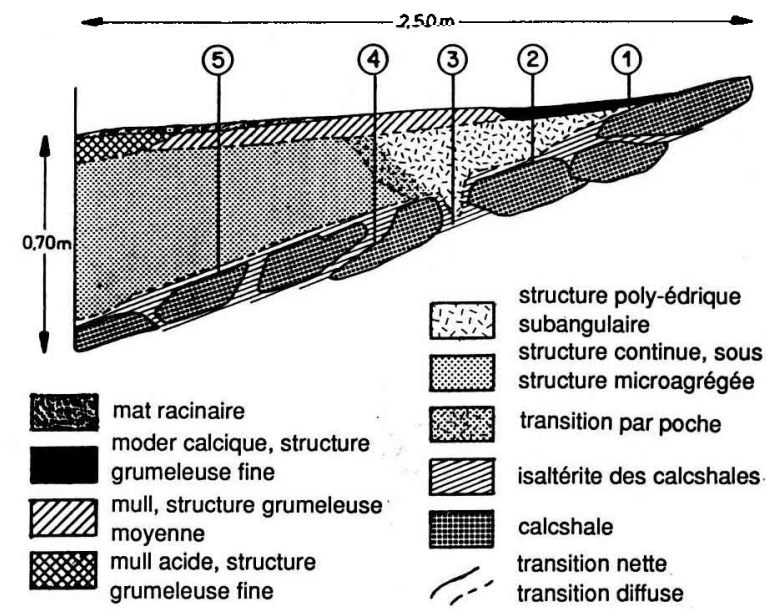

Fig 2. Présentation schématique d'une séquence courte située à proximité d'un affleurement des calcshales (les numéros indiquent l'emplacement des prélèvements de sol). 


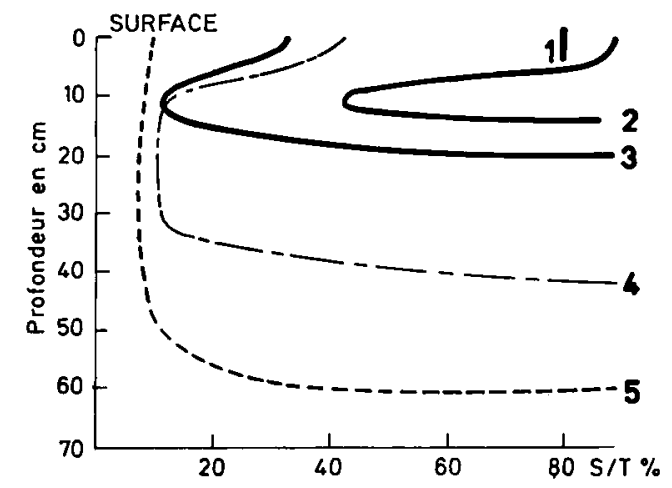

Fig 3. Évolution du taux de saturation $(\mathrm{S} / \mathrm{T})$ en fonction de la profondeur des sols, au voisinage d'un affleurement. (Les numéros des courbes correspondent aux profils repérés sur la séquence de la figure 2).

Au niveau des profils pédologiques, les indicateurs macroscopiques d'une telle évolution sont l'apparition d'un mat racinaire, l'évolution du type d'humus et de la structure (fig 2). On note en particulier la disparition de la structure polyédrique subangulaire et l'apparition d'une structure continue à tendance micro-agrégée (station 5). Comme nous l'avons montré, cette structure offre des propriétés physiques dévaforables : faible disponibilité de l'eau pour les racines, faible perméabilité pour l'eau et pour l'air. Par conséquent, on peut penser que c'est la présence de cet horizon $B$ à structure continue qui limite l'accès des végétaux aux abondantes réserves en eau et en bases de l'horizon d'altération.

Ainsi, dans ce type de milieu, l'approfondissement du sol, au-delà d'une certaine limite, n'améliore pas le régime trophique du couvert herbacé. Au contraire et en raison des propriétés spécifiques de l'horizon B, les fortes potentialités correspondent à des sols de profondeur moyenne $(20-30 \mathrm{~cm})$, c'est-à-dire aux situations qui autorisent l'établissement de relations racinaires entre les bonnes espèces fourragères et la roche en cours d'altération.

Ce type de fonctionnement n'apparaît que pour des pendages plus ou moins parallèles à la pente et sur des pentes relativement douces, conditions dans lesquelles l'évacuation des produits d'altération reste lente. Tout ceci souligne bien l'intérêt d'une étude attentive des couches intermédiaires sols-substrats pour un diagnostic du milieu végétal (Callot, 1978).

\section{Pratiques pastorales, état structural des sols et dégradation des herbages}

Pour caractériser les relations sols-pratiques d'entretien des herbages nous avons étudié 3 stations à sols profonds (I, II, III, voir fig 1), différant seulement, et ceci de longue date (> 30 ans), par le mode d'exploitation, la végétation et sa valeur d'usage (tableau II). Les autres caractéristiques des stations se rapportant à la topographie, au faciès de la roche mère et au matériau d'altération sont constantes.

Sur le plateau, la dégradation fourragère se marque surtout par l'extension des végétations maigres acidophiles peu appétantes, dominées par le nard et ses compagnes habituelles. En fait, la nardaie (station II) succède à la prairie grasse (à trisète, station I) après l'abandon des pratiques organisées de restitution de matière organique, à condition qu'une intensité minimale de pâture se maintienne. En effet, l'état herbacé est très instable dans ce type de milieu acid (Dorioz, 1987) et rapidement, les Ericacées telles que les myrtilles (station III) envahissent les pelouses soumises à de trop faibles pressions pastorales: Par la suite, en quelques décennies, on aboutit à une lande dont l'usage pastoral est quasiment nul.

Nos 3 stations s'organisent donc comme une séquence de dégradation de la couverture végétale. Nous nous interrogerons maintenant sur la transformations du sol associées à cette évolution.

\section{Caractérisation des différences structurales liées à l'exploitation}

Les granulométries confirment l'identité des matériaux d'origine. Les analyses chimiques ne mettent en évidence que des différences mineures en terme de $\mathrm{pH}$, taux de saturation, CEC; les courbes $\mathrm{pF}$ - teneur en eau montrent que les comportements hydriques sont assez similaires pour les valeurs de $\mathrm{pF}>1$ (Van Oort, 1984). II s'agit donc de matériaux très proches, offrant globalement les mêmes structures microscopiques et les mêmes capacités de réserve en eau.

En fait, les sols des 3 stations diffèrent surtout au niveau de leur état structural macroscopique. Ceci s'observe aisément sur le terrain et se traduit en lames minces (fig 4) par une nette diminution de la taille moyenne des unités de la sur- 


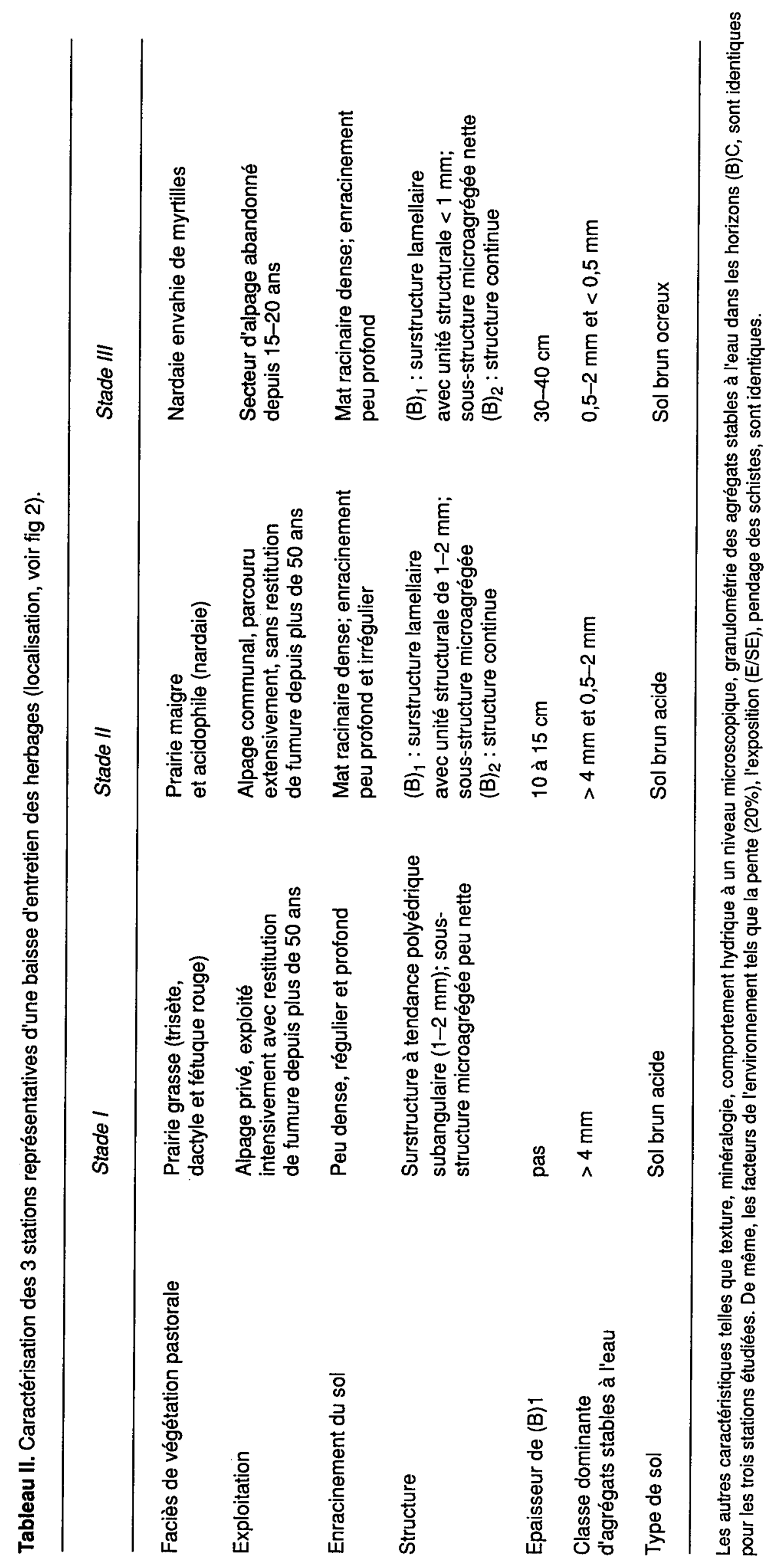



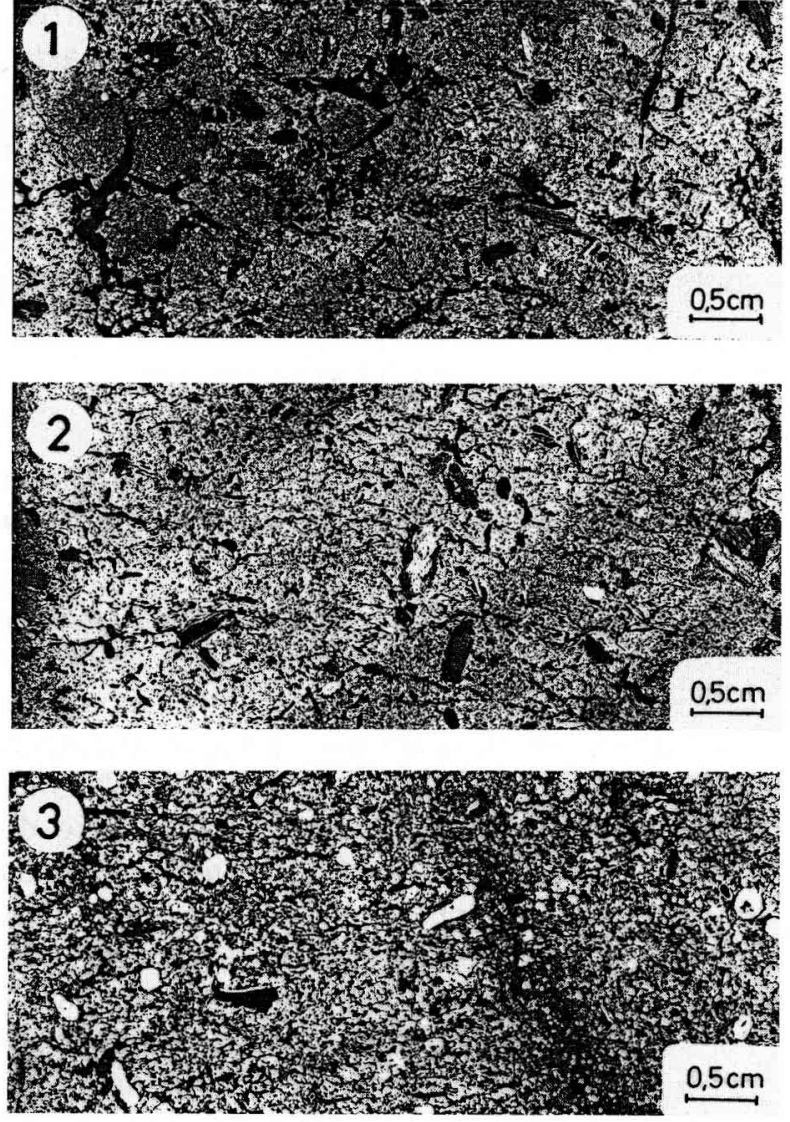

Fig 4. Observation sur lames minces de la structure dans les horizons (B). 1: station I; GX2; 2: station II; GX2; 3: station III, GX2 (dans les images présentées les vides $(V)$ apparaissent en noir).

structure dans l'horizon $B$ des stations sans restitution (II), puis une disparition de cette surstructure dans les stations abandonnées (III). Cette évolution a pu être quantifiée par la mesure de la distribution de la taille des agrégats stables à l'eau (tableau II). Par conséquent, en terme de porosité, ces changements de structure correspondent à l'apparition d'une macroporosité continue (III) qui coïncide avec une augmentation des possibilités d'écoulement rapide de l'eau.

\section{Interprétation de la séquence de dégradation}

Les modifications de la végétation et de l'activité pastorale se traduisent d'abord par un changement qualitatif et quantitatif de la litière restituée. On passe d'une litière herbacée enrichie en azote par les déjections, à une litière comprenant des débris ligneux de décomposition difficile. L'activité biologique du sol étant globalement responsable de l'agrégation et de la stabilité structurale des agrégats (Hénin, 1976; Tisdall et Oades,
1982; Dorioz et Robert, 1987), c'est bien le changement des pratiques pastorales qui est à l'origine des transformations structurales et porales du sol.

La structure polyédrique de la station I à bonne valeur pastorale, correspond à une bonne incorporation de la matière organique en relation avec une activité biologique intense, stimulée par l'activité pastorale (Gachon et al, 1979). Bien entendu, ceci se manifeste par un meilleur état trophique des sols $(\mathrm{C} / \mathrm{N}$ plus bas; $\mathrm{K}$ échangeable 10 fois plus élevé...), par une meilleure exploration racinaire et une dynamique de l'eau plus favorable. Or, sur ce matériau limoneux, une telle structure représente un état instable et, avec la baisse des restitutions fertilisantes, une évolution s'amorce : la taille des éléments structuraux se réduit, d'abord dans la partie supérieure de l'horizon (B), puis ensuite vers la profondeur. Les possibilités d'écoulement de l'eau dans le profil s'accroissent, surtout quand ce processus de restructuration atteint l'horizon $B(C)$ lui-même très poreux dès l'origine. L'aptitude au drainage (Cabidoche, 1979) globale verticale du profil augmente alors considérablement. Ainsi modifié, ce matériau déjà acidifié, permet l'écoulement de grandes quantités d'eau, entraînant les divers éléments en solution, ce qui favorise la podzolisation. D'ailleurs sur des sols bruns acides également sur calcschistes, Dambrine (1985) enregistre des fonctionnements géochimiques saisonniers équivalents à ceux de podzols. Le podzol, stade ultime de cette évolution s'observe en bordure du plateau de référence, sous lande à rhododendron (fig 1).

\section{Généralisation : fonctionnement des unités de plateau sur calcshales}

En position de plateau, l'horizon (B) microporeux se comporte, en été, comme un matériau sec génant le fonctionnement du système racinaire, isolant les végétaux des réserves d'eau situées dans l'altérite (C). Ceci n'empêche pas que ce matériau soit fortement désaturé en raison des percolations intenses et lentes se produisant à la fonte des neiges. Au plan pratique ces phénomènes limitent sérieusement les potentialités fourragères de sols par ailleurs profonds et sains.

En terme de pédogénèse, la tendance générale de l'évolution de ces sols situés à l'étage 
subalpin se rapporte à la voie podzolique (acidocomplexolyse). Dans le détail, cette évolution se manifeste plus ou moins clairement selon l'aptitude au drainage des profils. Or, dans notre cas ce paramètre qui contrôle la lixiviation dépend de la structure du sol, elle-même très soumise à l'entretien pastoral. Cet effet de l'exploitation sur le sol explique que, selon l'intensité des restitutions, on puisse observer, à quelques mètres l'un de l'autre, des sols bruns modaux bien structurés et des sols podzoliques. Les premiers, en raison d'un entretien séculaire (Bouma et al, 1969), portent des herbages de bonne valeur. Mais cet équilibre sol-potentialité est fragile et se rompt en quelques années d'abandon du fait des caractéristiques physiques défavorables du matériau. Les podzols qui en résultent sont en pratique irrécupérables pour l'agriculture actuelle (Dambrine, 1981).

Au total, en situation de plateau, les propriétés physiques des matériaux d'altération et leurs interactions avec la pédogénèse et sa tendance à la podzolisation, déterminent les contraintes d'usage des terres. À l'exception de rares affleurements calcaires, les meilleures potentialités de ces secteurs correspondent aux terrains entretenus intensément et de longue date. Pour comprendre l'organisation végétale de ce type de paysage il est indispensable de se référer à l'histoire de l'exploitation des diverses unités pastorales.

Quand la pente s'accentue, les facteurs liés à l'exploitation perdent de l'importance; un autre fonctionnement typique apparaît : celui des versants de solifluxion.

\section{LES UNITÉS DE VERSANT SOLIFLUÉ}

La zone de référence (600 ha entre 1700 et $2200 \mathrm{~m}$ d'altitude) s'étend sur les versants d'une montagne de calcschistes (fig 5).

Nous avons porté une attention plus particulière à la toposéquence du versant à pendage conforme, toute entière incluse dans la même unité pastorale et exploitée de façon intensive. Les pratiques se différencient peu selon l'axe altitudinal en raison de la forme rectangulaire de l'alpage, de l'emplacement des divers bâtiments et surtout de la recherche, par l'exploitant, d'un découpage rationnel de l'espace (parcs avec rotations assez régulières). Par ailleurs, les restitutions organiques sont favorisées au maximum (déplacements des parcs de nuit et de traite, épandage des bouses...). Bien entendu, malgré cette homogénéité des pratiques à l'échelle de

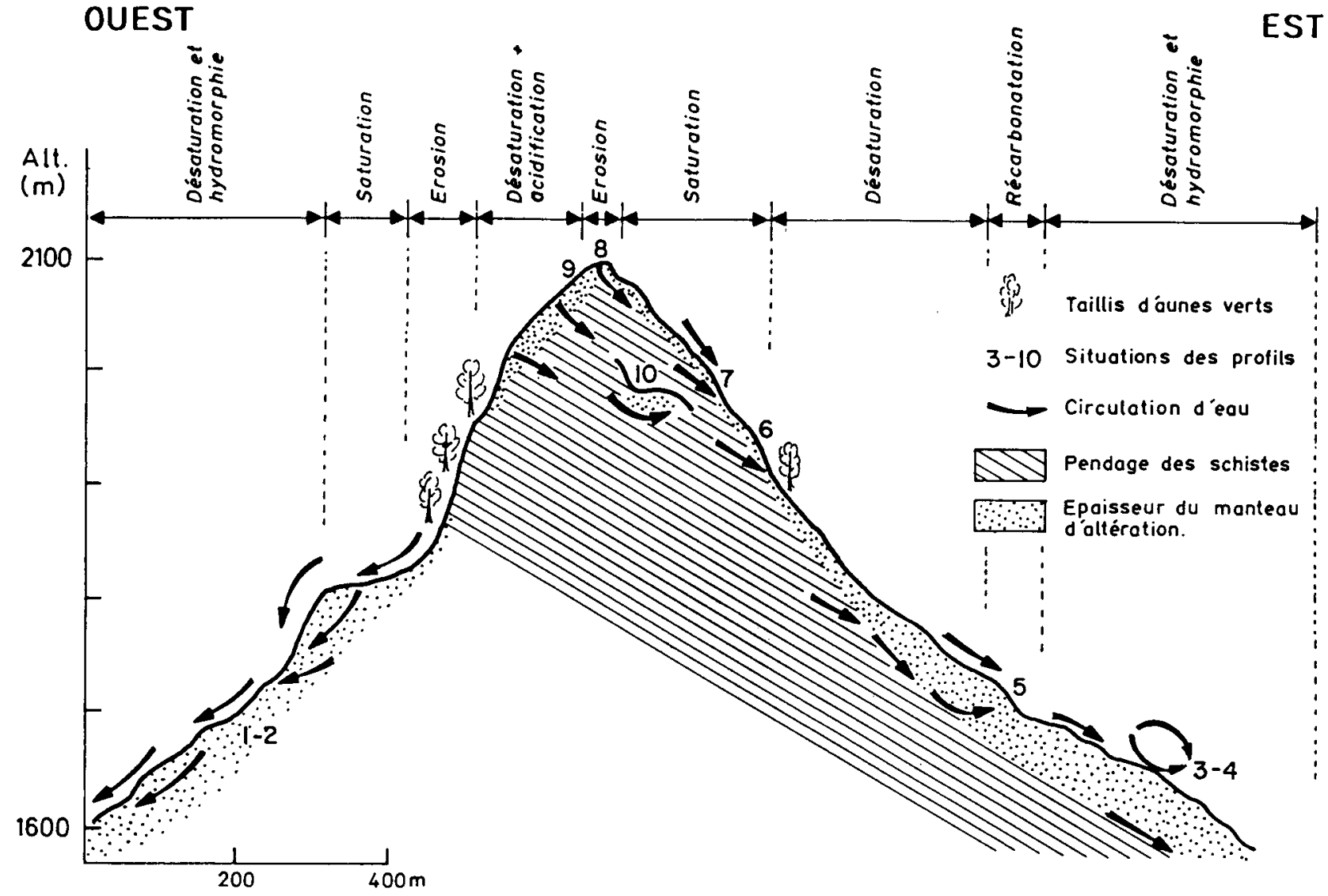

Fig 5. Versant sur calcshistes : toposéquence, circulation de l'eau et différenciation de la couverture pédologique (les $n^{\circ}$ font référence aux stations étudiées en détail, voir texte). 
l'alpage, il existe des surfaces périphériques, plus marginales, fréquentées et entretenues de manière beaucoup plus extensive. On dispose finalement d'un bon modèle pour analyser, en conditions de versant, les interactions solsvégétations-exploitations.

\section{Vue d'ensemble sur l'organisation de la toposéquence}

Grâce aux observations morphotactiles de terrain et aux analyses de sol (tableau III) on peut mettre en évidence les caractères généraux définissant la toposéquence étudiée.

\section{Bonne homogéneité texturale des sols}

Les caractéristiques texturales des profils correspondent bien à l'évolution typique des sols issus de l'altération des calcschistes, sauf en position de crête ou de cuvette.

\section{Augmentation du taux de matière organique}

On note une augmentation globale du taux de matière organique vers le haut du versant, dans les sols non hydromorphes : il peut s'agir d'un effet dû au gradient climatique.

\section{Augmentation de la profondeur des sols}

La profondeur des sols augmente, en moyenne, vers le bas du versant; seules les situations sommitales et quelques ruptures de pente offrent des sols superficiels $(20-30 \mathrm{~cm})$. Parallèlement au pied du versant, la surface occupée par les zones hydromorphes est particulièrement étendue. Ces faits s'expliquent par une plus grande épaisseur du matériau soliflué et par une plus grande quantité cumulée d'eau.

II apparaît donc d'emblée que les phénomènes liés à la géomorphogénèse et à la circulation superficielle de l'eau sont les facteurs d'organisation majeurs de cette unité.

\section{Circulation de l'eau dans les matériaux superficiels, organisation des sols et des faciès de végétation}

La circulation de l'eau ne se résume pas à une simple accumulation en bas de pente et dans les dépressions. Si l'on considère les variations du $\mathrm{pH}$ et de $\mathrm{S} / \mathrm{T}$ (tableau III) de bas en haut du ver- sant, on remarque la présence d'un secteur calcique et même parfois carbonaté (profils 6,5 et 7) à mi-pente. Cette position correspond, sur le terrain, à des niveaux de sortie diffuse d'eau avec localement des reprécipitations de carbonates. Les sols prennent parfois des caractères hydromorphes (profil 7). La végétation herbacée apparaît toujours à la fois exubérante et nettement calcicole.

À l'inverse, sur les replats relatifs de ce versant (profil 10) on observe des prairies maigres (nardaies) sur des sols acidifiés. La même situation prévaut immédiatement en contrebas de la crête sur le versant inverse à schistosité perpendiculaire à la pente (profil 9). Dans ces 2 types de stations, l'eau exerce une action appauvrissante et favorise l'existence de sols acides.

En bas de versant, les solifluxions créent un relief bosselé. Cette morphologie s'accompagne d'une circulation préférentielle de l'eau en surface du sol et d'une distribution en mosaïque des sols à une échelle décamétrique. On observe alors toute une gamme de stations exprimant un gradient d'hydromorphie allant du sol brun "sain" au sol à pseudogley et au sol tourbeux sur gley (profils 3 et 4). La végétation, si elle répond bien à cette différenciation très fine, n'en reproduit pas pour autant tous les détails en raison de l'action des facteurs d'exploitation. La flore typique des marécages (Caricion davallianae), négligeable au plan de la valeur pastorale, forme des taches très nettes, localisées uniquement sur les sols très humides. En fait, l'apparition d'un horizon brunifié superficiel de $5-10 \mathrm{~cm}$ suffit pour permettre l'installation de prairies grasses de très bonne valeur (Triseto - polygonion ou Poïon alpinae). Cet état est facilement obtenu dans la plupart des dépressions par un drainage sommaire (réseau de petits fossés). Sur les marges de l'alpage, loin des bâtiments, ce type d'entretien a été abandonné depuis une décennie : la surface marécageuse s'est étendue rendant impossible le parcage nocturne des animaux. On observe alors diverses évolutions du tapis herbacé, avec en particulier installation de quelques $\mathrm{m}^{2}$ de pelouse maigre à nard sur certaines bosses. Cette dégradation n'apparaît que dans les stations suffisamment surélevées. En position plus basse, l'influence de l'eau circulant sur le versant limite la dégradation pastorale de la végétation. L'extensification de l'exploitation agit à nouveau comme un révélateur des relations entre dynamique végétale, contraintes d'entretien des sols et paramètres topographiques. 


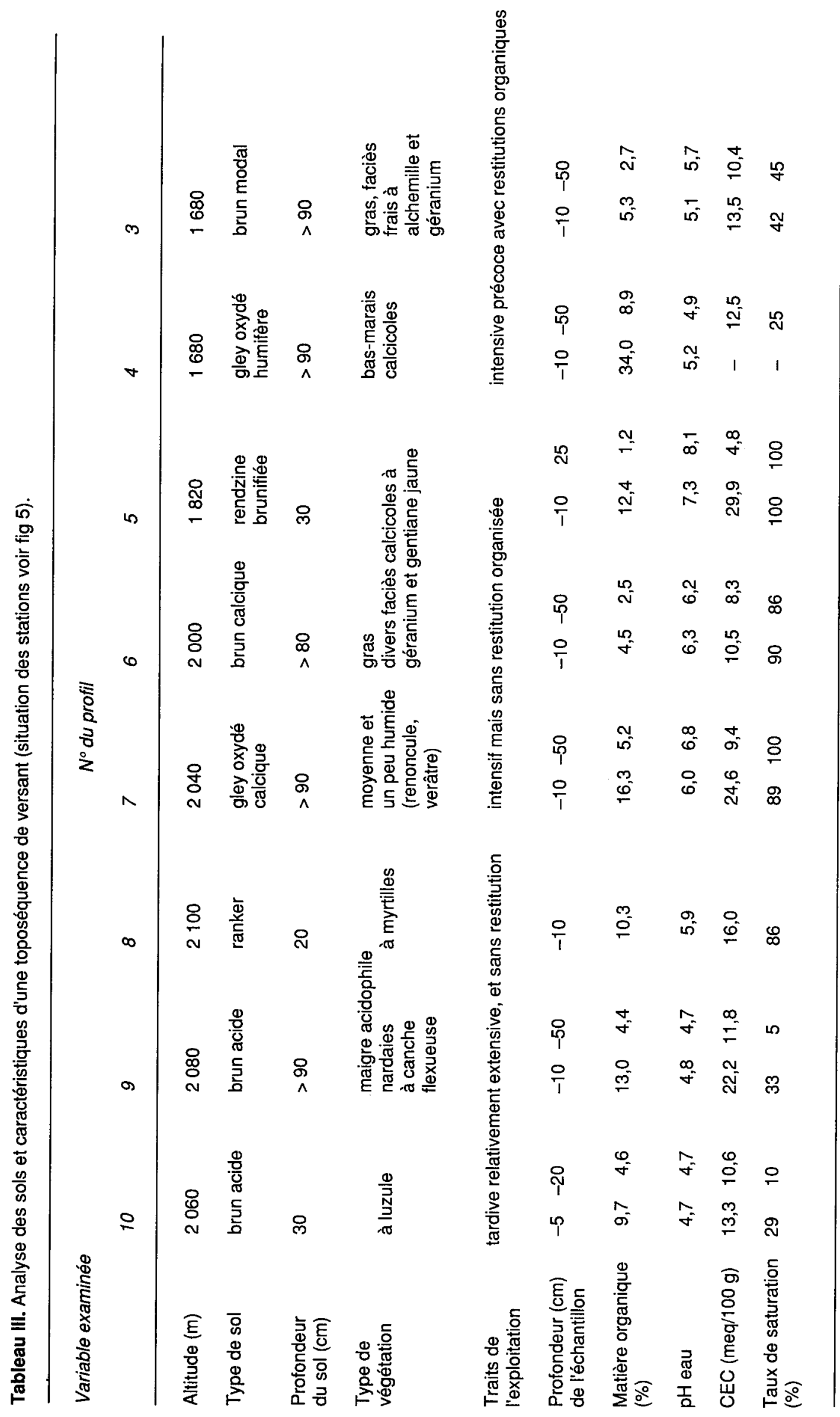




\section{Modèle de fonctionnement des unités de versants}

Tous ces éléments nous amènent à proposer un modèle schématique de l'organisation agroécologique des versants sur calcschistes (fig 5, tableau IV).

Quel que soit le versant considéré, l'évolution des propriétés des sols est étroitement liée à leur position topographique. Le relief agit pour l'essentiel en contrôlant la quantité et la qualité de l'eau distribuée dans le milieu. Par comparaison, les effets du gradient climatique apparaissent secondaires malgré une dénivelée de $500 \mathrm{~m}$.

Si l'on compare les 2 versants (tableau V) d'une même montagne, il est nécessaire de prendre en compte le pendage des calcshales et ses conséquences sur la circulation superficielle de l'eau, pour expliquer les différences d'exploitabilité, de potentialité fourragère. Le pendage induit d'abord une dissymétrie géomorphologique et pédologique qui s'exprime tout particulièrement entre la crête et la mi-pente du versant.

Le versant à schistosité perpendiculaire est plus accidenté. II présente à la fois des secteurs de pentes plus fortes largement colonisés par les aulnes verts et des replats relativement vastes (1 ha) dus à quelques glissements en masse. Cette topographie favorise l'éclatement des alpages en sous-unités plus ou moins isolées et exploitées avec des intensités très variables. Par ailleurs, la partie haute de ce versant est constituée de milieux à sols acides en raison d'une action essentiellement appauvrissante de l'eau. II s'agit là de situations pastorales peu favorables, à végétations herbacées maigres, avec une tendance à l'évolution rapide vers la lande à Ericacées. En général, cette partie du versant est sous-exploitée ou en cours d'abandon, soit à la fois en raison de son trop faible potentiel et de son exploitation trop tardive.

Le versant à pendage conforme offre une situation bien différente, avec une topographie généralement plus régulière, les pentes se redressant progressivement vers le haut. II constitue aussi un milieu à ambiance calcique, fraîche, à forte potentialité, le tout favorisant le maintien de l'exploitation pastorale. En effet, grâce à un "effet tampon» de l'eau rechargée en carbonates et au remaniement dû à la solifluxion, les sols n'évoluent pas au-delà du stade sol brun acide. La valeur pastorale des herbages reste très généralement favorable, même en l'absence d'entretien de la fertilité. Pour maintenir un état pastoral correct, il suffit en fait d'une pression pastorale minimale et d'un entretien du drainage.

Les bas des versants, indépendamment du pendage des roches, sont soumis à ces effets tampons liés aux transferts par l'eau. La solifluxion aboutit à un paysage bosselé constitué par une mosaïque de milieux à sols plus ou moins hydromorphes, potentiellement très productifs, avec une bonne repousse après pâture. Les quelques mesures de production de matière sèche réalisées révèlent en moyenne 3 fois plus d'herbe disponible que dans le milieu acide, décrit précédemment (Sabin, 1987). Les estimations qualitatives (indices de valeurs pastorales), confirment l'aptitude à la productivité de ce type de milieu. Cependant, ces bons résultats sont liés au maintien d'un système de drainage sans lequel on aboutit à une extension des bas marais et des taillis à aulnes verts, ainsi qu'à une concentration des activités de pacage sur les parties restées saines. Ces dernières évoluent alors fréquemment vers des prairies envahies par le vérâtre et/ou par le rumex des Alpes, espèces dont l'extension constitue le risque majeur de dégradation dans ce type d'alpage.

\section{CONCLUSION}

Dans le Beaufortain, comme dans d'autres régions alpines (Dorioz, 1987), la valeur d'usage des pâtures d'altitude (appétence, contraintes d'entretien, productivité...) repose pour une large part sur des paramètres liés aux facteurs édaphiques. Bien entendu, d'autres paramètres interviennent également (exploitabilité, éloignement, précocité relative...), mais globalement, le sol constitue un élément explicatif majeur.

Les paramètres édaphiques actifs et les modalités des interactions sol-exploitation, diffèrent selon la place de la station dans le paysage.

En position surélevée, plateau, le matériau et les tendances de la pédogénèse sont peu favorables à l'herbe; les ressources fourragères dépendent d'abord de l'entretien pastoral. Le paysage est organisé en unités pastorales. Les risques de dégradation par extensification sont les plus élevés, les divers stades de déprise étant conditionnés par les propriétés des sols, leur épaisseur et l'histoire des stations.

Sur les versants, les conditions sont radicalement différentes. Les apports de l'amont, en particulier d'eau, limitent les risques d'appauvrissement des sols (effet tampon), et constituent le trait le plus marquant de l'écologie de ces paysages organisés en unités géomorphologiques. 


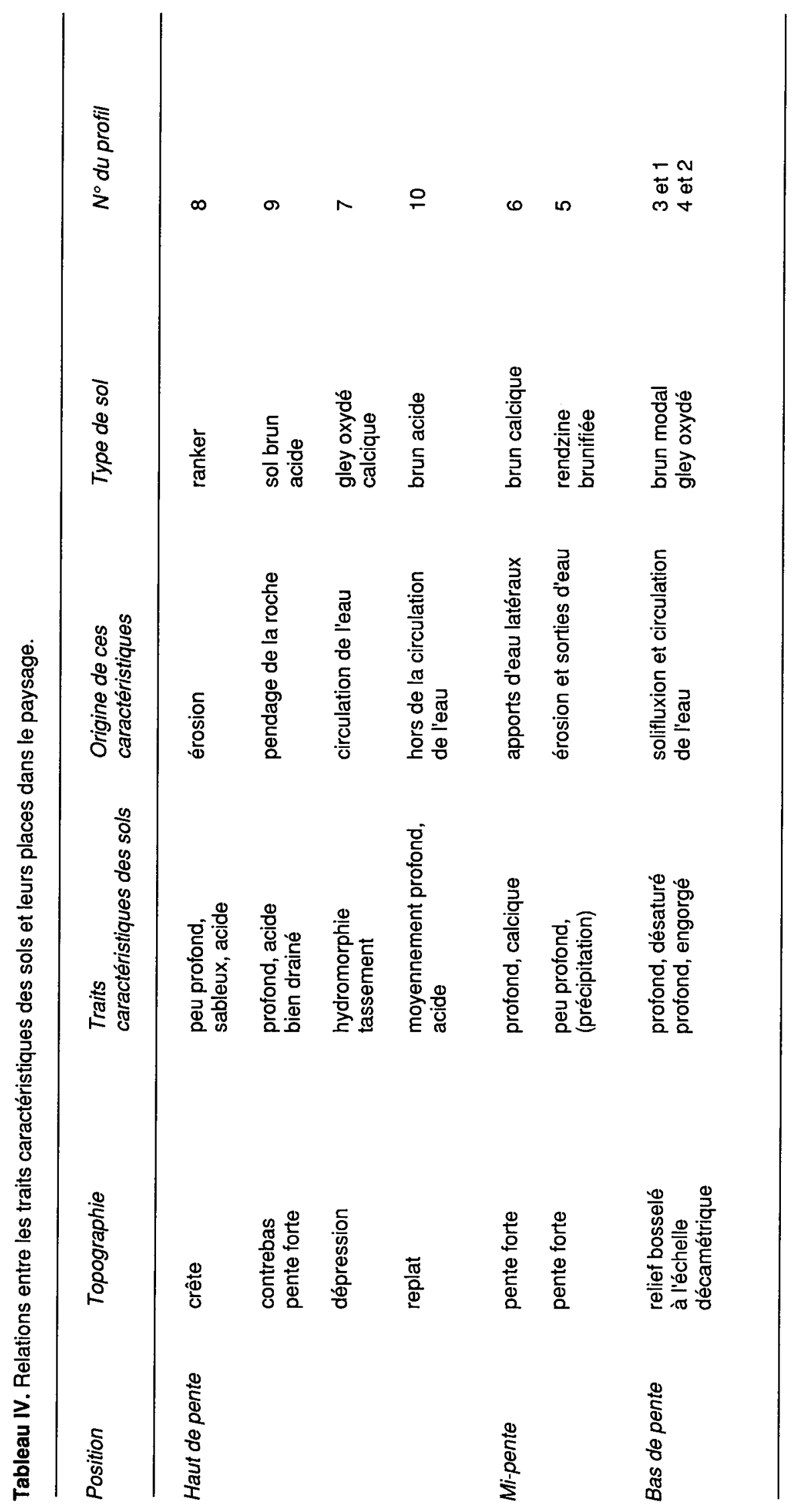


Tableau V.

\begin{tabular}{|c|c|c|}
\hline Position & $\begin{array}{l}\text { Schistosité perpendiculaire } \\
\text { à la pente }\end{array}$ & $\begin{array}{l}\text { Schistosité parallèle } \\
\text { à la pente }\end{array}$ \\
\hline
\end{tabular}

Pente régulière

Haut de pente

Mi-pente

Exploitabilité générale en eau

Bas de pente Mosaïque de sols bruns modaux et de sols hydromorphes

\begin{abstract}
Sols bruns acides, profonds
\end{abstract}
Herbages peu appétants, dominés par des plantes acidophiles

En cas d'abandon, évolution rapide vers landes à myrtille

Falaises ou pentes très raides avec forêt d'épicéa ou taillis d'aulnes verts

\section{Relief irrégulier}

Sols bruns calciques ou bruns calcaires

Herbages de bonne qualité à base de graminées calcicoles

Moins sensible à la dégradation par les ligneux

Sols bruns calciques et calcaires, ou sols hydromorphes

Herbages de bonne qualité à base de graminées calcicoles (type moyen ou gras)

Sols profonds, développés dans une couche épaisse de solifluxion, en général bien alimentés

Nombreuses formations végétales : du bas marais à la prairie grasse fraîche à trisète

Très forte production

Surface exploitée morcelée pour le haut et le bas de pente
Surface exploitable de haut en bas d'un seul tenant
En fait, dans le détail, 2 phénomènes liés à la pédogénèse sont déterminants et significatifs au plan agronomique, dans le secteur des calcshales.

\section{La décarbonation}

On peut opposer en termes agronomique et phytosociologique les milieux à sols acides et les milieux à sols calciques ou calcaires. Les premiers offrent une herbe généralement plus appétante, plus productive et surtout moins exigeante en entretien. Les seconds sont d'un usage pastoral moins souple en raison de leur facilité à évoluer vers des communautés végétales défavorables, en cas de charge pastorale insuffisante. En fin de compte, la compréhension du fonctionnement physico-chimique du sol contribue à la prévision de l'évolution des ressources fourragères, en particulier en cas d'extensification.

\section{Le fonctionnement hydrique}

La fourniture hydrique estivale règle l'état des ressources fourragères en fin de saison. Or, malgré la forte pluviométrie, de nombreux sols de montagne lorsqu'ils sont placés sur des points hauts ont un comportement hydrique défavorable pour la végétation, malgré leur profondeur et leur très faible charge en éléments grossiers. Ils s'opposent très nettement aux sols souvent très caillouteux mais alimentés continuellement par des transferts latéraux (bas de versant soliflué, mi-pente des versants à pendage conforme).

L'importance agronomique de ces phénomènes se manifeste aussi bien à l'échelle régionale (opposition entre les aptitudes des versants et de plateaux) qu'à l'échelle d'une station (relation profondeur du sol - valeur de l'herbe). La connaissance de leurs lois de répartition fournit donc un modèle de compréhension du milieu di- 
rectement opérationnel à l'échelle de l'unité pastorale et de la région.

Les milieux calcschistes et les matériaux voisins étant largement représentés dans les Alpes, l'expérience acquise peut être transposée à de vastes surfaces pastorales développées sur des roches sédimentaires plus ou moins calcaires, et sur les matériaux issus de leur érosion. Dans de telles conditions, si l'on cherche à comprendre les aptitudes agropédologiques d'une région, la stratégie suivante peut être proposée :

- caractérisation de la valeur des herbages (par enquête);

- étude des règles de répartition des milieux calcaires, calciques et acides dans le paysage (Legros et al, 1987). De ce point de vue, dans le milieu calcshale et schiste une attention particulière doit être portée aux pendages, à la position topographique; à l'épaisseur des sols, à la présence d'éléments grossiers colmatés;

- analyse des propriétés intrinsèques du matériau d'altération à partir de sols en place. II est important d'insister sur les facteurs explicatifs des propriétés physico-chimiques : minéralogie des constituants, CEC, garniture cationique, trajet granulo-dynamique (Legros, 1982), origine de la géométrie et de l'évolution du système de porosité (van Oort, 1984; Chrétien et Pedro, 1987). En montagne, sous climat humide, ces propriétés conditionnent l'aptitude des matériaux pédologiques à "tamponner», indépendamment des pratiques d'exploitation, les évolutions défavorables des systèmes sol-végétation, qu'il s'agisse d'évolution à long terme (acidificationpodzolisation) ou d'évolution saisonnière (sécheresse estivale, tassement, excès d'eau);

- caractérisation des facteurs hydriques au niveau du profil, mais surtout au niveau du versant.

\section{REMERCIEMENTS}

Cet article a bénéficié des conseils de Mrs JP Legros (INRA Montpellier) et A Bruand (INRA Orléans). II a été réalisé en grande partie dans le cadre du programme de recherche développement sur l'agriculture des Alpes du Nord.

\section{RÉFÉRENCES}

Bouma J, Hoeks J, Van der Plas, Van Scherrenburg BJ (1969) Genesis and morphology of some alpine podzol profiles. J Soil Sci Vol 20, $n^{\circ} 2,384-398$
Cabidoche G (1979) Contribution à l'étude des sols de haute montagne. Analyse des relations sols-milieux dans les Pyrénées Occidentales, cristallines et calcaire. Thèse Doc Sp, Univ Montpellier, $158 \mathrm{p}$

Callot $G$ (1978) Analyse des litho systèmes carbonatés. Rôle du substratum calcaire dans la pédogénèse. DOC - SES - INRA - Montpellier, $n^{\circ} 484,23 p$

Chrétien J, Pedro G (1987) Granulométrie, porosité et spectre poral des sols développés sur formations détritiques. Cas des terrasses alluviales de la Saône. Cahiers de l'ORSTOM, XXIII (1), 43-54

Dambrine $E$ (1981) Répartition des phénomènes pédologiques sur le plateau des Saisies. DEA Paris $\mathrm{VI}, 90 \mathrm{p}$

Dambrine $E$ (1985) Contribution à l'étude de la répartition et du fonctionnement des sols de haute montagne. Thèse Univ Paris VII, $284 p$

Dejou J, Guyot J, Pedro G, Chaumont C (1971) Étude sur le rôle du pendage dans l'évolution superficielle des roches schisteuses. Cas de l'altération de micaschistes dans le Massif central français. Pédologie XXI, 3, 343-359

Dorioz JM (1987) Dynamique écologique des territoires pastoraux des Alpes du Nord. 2. Acta Oecol, Oecol App/ 8, 257-280

Dorioz JM, Party JP (1987) Dynamique écologique et typologie des territoires pastoraux des Alpes du Nord. 1. Analyse de l'organisation agroécologique d'un secteur de référence. Acta Oecol Oecol Appl $6,257-280$

Dorioz JM, Robert M (1987) Aspects microscopiques des relations entre microorganismes ou végétaux et les argiles. Conséquences sur les microorganisations et la microstructuration des sols. In: Micromorphologie des sols (N Fedoroff, LM Bression, MA Courty, ed), Paris, 1985, 521-528

Dubost M, Bornard A (1989) Typologie des végétations d'alpage. Document GIS Alpes du Nord, Chambéry, $20 \mathrm{p}$

Gachon L, Ricou G, Gruner L (1979) Fonctionnement de l'écosystème prairial pâturé. In: Utilisation des pâturages d'altitude et des parcours méditerranéens (INRA). Versailles, 9-19

Hénin S (1976) Cours de physique du sol. Tome I. ORSTOM - Editest, Init-Doc Tech, $\mathrm{n}^{\circ}$ 29, 159 p

Jeannin B, Fleury P, Dorioz JM (1991) Typologie des prairies de fauche des montagnes des Alpes du Nord : principes et applications. Fourrages (à paraître

Legros JP (1982) L'évolution granulométrique au cours de la pédogenèse. Approche par simulation sur ordinateur. Thèse doc d'état, Univ Montpellier, $436 \mathrm{p}$

Legros JP, Robert M, Van Oort F (1980) Caractères principaux de la pédogénèse sur calcschiste en montagne. Sci Sol 4, 297-312

Legros JP, Party JP, Dorioz JM (1987) Répartition des milieux calcaires, calciques et acidifiés en haute montagne calcaire humide. Conséquences agrono- 
miques et écologiques. Doc Carto Ecol, Grenoble, vol XXX, 137-157

Nicot $J$ (1988) Le Beaufortain oriental. Présentation d'une carte géomorphologique et géodynamique au 1/30.000. Revue de Géo alpine. t LXXVI, 121-146

Reydet V (1985) Étude du «fonctionnement" écologique de deux alpages du Beaufortain. DEA, Chambéry, $40 p$

Richard L, Pautou G (1982) Carte de la végétation au 1/200 000 Alpes du Nord et méridionales. CNRS, Paris, $316 \mathrm{p}$

Sabin J (1987) Production laitière en alpage. Recherche sur le pâturage et la production. ISA. Chambre Agriculture de Savoie. Chambéry, 76 p + annexes
Tisdall M, Oades JM (1982) Organic matter and water stable agregates in soils. J Soil Sci 33, 141-163

Tricart F, Killian F (1979) Écogéographie. Hérodote Maspero, Paris, $230 \mathrm{p}$

Van Oort F (1984) Géométrie de l'espace poral, comportement hydrique et pédogénèse. Application à des sols sous prairie et alpage, issus de l'altération des calcschistes sédimentaires en moyenne et haute montagne, Alpes du Nord (Beaufortain). Thèse Doc Ing INA-PG, $281 p$

Van Oort F, Dorioz JM (1991) Écologie des paysages pastoraux alpins de la zone des calcschistes sédimentaires (Beaufortain). I. Caractères de l'altération, propriétés hydriques et porales associées. Agronomie 293-303 